\title{
Envejecimiento, lesbianismo y heteronormatividad en la película 80 egunean
}

(2010).

\section{Raquel Medina \\ Aston University}

A pesar de que el envejecimiento demográfico es un fenómeno global, el cine como producto cultural y barómetro de la realidad apenas captura este fenómeno ni lo hace visible. En las culturas occidentales, los cuerpos envejecidos no existen, se ocultan, con lo que están sujetos al estigma y la exclusión (Casper and Moore 2009). La discriminación que el envejecimiento sufre en el cine, sobre todo el envejecimiento positivo, es aún mayor cuando los sujetos son mujeres y/o no son heterosexuales. En este sentido, la película de Inés París y Daniela Féjerman, A mi madre le gustan las mujeres (2002), puede considerarse una excepción, a la vez que pionera en España, al tratar la salida del armario de una mujer de unos sesenta años, separada y madre de tres hijas ya adultas, que mantiene una relación con una mujer mucho más joven que ella ${ }^{1}$. Sin embargo, tendrán que pasar nueve años hasta que dos directores vascos, Jon Garaño y Jose Mari Goenaga, estrenen 80 egunean -En 80 días--(2010), una película que plantea la relación sentimental y sexual entre dos mujeres de setenta años: una de ellas abiertamente lesbiana y otra casada que, hasta el encuentro con su amiga de la adolescencia, creía ser heterosexual. El presente artículo tiene como objetivo analizar la relación de estas dos mujeres en 80 egunean a través de la intersección de género, sexualidad y edad para así entender este texto fílmico como el lugar de encuentro de todas estas cuestiones. Dentro de este análisis también se estudiarán la identidad sexual y la edad como aspectos entrelazados que no pueden

\footnotetext{
${ }^{1}$ Hay una larga tradición en el cine español de a partir de los 70 de presentar relaciones gais entre un hombre maduro y un joven efebo (Mira 2008)
} 
desvincularse entre sí en la película, así como que la noción de identidad sexual no se presenta en su cualidad nómada (Braidotti 1994) como cabría esperar en una película de temática homosexual, sino como una fija, que nunca llega a transformarse. La relación entre las dos mujeres que forma el foco narrativo tiene lugar espacial y temporalmente en ámbitos muy específicos que remiten simbólicamente, como se verá, a una exploración profunda de la edad, la sexualidad y los papeles de género, e incluso de la familia y de la propia identidad vasca. ${ }^{2}$ Sin embargo, como se dilucidará con el análisis, la heteronormatividad y la mirada masculina entendidas como las relaciones de poder por medio de las cuales las relaciones heterosexuales se institucionalizan, tal y como enfatizó Michael Warner (1991), serán las que prevalezcan finalmente. Aunque la cinta pone de manifiesto la supuesta “normalización" de la homosexualidad en el paisaje social vasco, no establece, como el análisis desvelará, un espacio y un tiempo queer ${ }^{3}$.

En su libro La vejez Simone de Beauvoir (1983) no únicamente pone de manifiesto cómo la sociedad percibe, representa y margina a las personas mayores, sino cómo ve y siente el envejecimiento el individuo, especialmente la mujer. Los discursos sociales hegemónicos han construido un concepto de vejez caracterizado por la pasividad, la decrepitud, la fragilidad y la improductividad. Por eso el vocablo

\footnotetext{
${ }^{2}$ No es mi propósito discutir aquí la existencia o no de un cine vasco, algo que ya han hecho otros: Gabilondo 2014; Rodríguez 2002; Rodríguez and Stone 2015; Roldan Larreta 1999; Torrado Morales 2008; De Pablo 2012; Zunzunegui 1985). . Tan solo indicar que la película se rueda en euskara y todo el equipo es vasco. . 80 egunean puede analizarse también desde el marco de la identidad vasca. . No es el propósito del presente ensayo, pero la división ciudad/campo, Donostia/caserío, los conflictos intergeneracionales y la revisión de la masculinidad pueden evaluarse en esta película en diálogo con películas vascas anteriores que problematizan estos aspectos en relación al nacionalismo vasco y la masculinidad (Gabilondo 1997; Stone and Jones 2004; Martín-Estudillo 2007; Davis 2009; Aresti 2014) .

${ }^{3}$ Emplearé el término queer en vez de las traducciones al castellano empleadas por otros como estudios torcidos o invertidos.
} 
en sí mismo se ha cargado de connotaciones negativas que necesariamente hay que eliminar. Los discursos en torno al envejecimiento, por tanto, han causado la discriminación de los mayores a través de la creación y sustentación de narrativas culturales que homogenizan y cargan de negatividad el envejecimiento e impelen al sujeto a un constante intento por enmascarar o disimular sus señales en el cuerpo (Cole et al. 1993; Featherstone 2000; Gullette 2004; Katz 1995; Woodward 1991). De este modo, tal y como indica Margaret Cruikshank (2008), el cuerpo se ha convertido en el texto en el que la edad se inscribe y se presenta, obliterando de este modo cualquier expresión que no sea de decadencia o de su intento por enmascararla. Los estudios etarios como estudios culturales se han visto reforzados en estas dos últimas décadas por los análisis feministas en torno a la edad y ponen asimismo de relieve la interseccionalidad de cuestiones de edad, género, sexualidad, religión, nacionalidad, etc., que en los textos culturales crean niveles discriminatorios entretejidos e interrelacionados. En este sentido, Margaret Morganroth Gullette (2004) analiza en el espacio cultural estadounidense cómo las diversas etapas de la edad se han convertido en herramientas discriminatorias que, a su vez, afectan más a las mujeres. Por su parte, Kathleen Woodward (1999) también ha explorado cómo esa discriminación de los mayores afecta a las mujeres de manera múltiple, pero sobre todo cómo la discriminación se centra especialmente en el cuerpo femenino desde la mirada masculina.

El cine como texto cultural ha sido uno de los ámbitos de representación cultural que ha omitido con mayor prominencia la presencia de personas mayores como protagonistas de sus narrativas. Sin embargo, cuando lo ha hecho, ha sido para reforzar los estereotipos en torno a la edad en los que prevalece un canto a la juventud, o para discriminar a la mujer de edad como pasiva y presentar a los héroes 
varones como todavía activos (Chivers 2011; Cabré 2014.). Pocas son, por lo tanto, las narraciones cinematográficas que se centran en el envejecimiento femenino como positivo y activo, y menos aún aquellas que presentan la sexualidad de los mayores ya sea ésta heterosexual u homosexual. El cine en España no ha sido una excepción; es más, este vacío ha sido enorme, con lo que pocas han sido las películas en las que las personas mayores son protagonistas y muchas menos en las que la sexualidad y las cuestiones de género se planteen abiertamente. La vida empieza hoy (2010), dirigida por Laura Mañá, puede considerarse como la única película española que introduce al espectador en el dormitorio y en la cama de dos personajes de más de 60 años.

Generalmente las relaciones sexuales explícitamente presentadas en la pantalla se dan cuando las protagonistas femeninas tienen menos de 45-50 años, por lo que la equiparación entre juventud/madurez y sexualidad se hace explícita en la pantalla y construye un discurso que niega la sexualidad de los mayores. Peor es todavía cuando hablamos del envejecimiento de LGBT en el llamado cine queer español ${ }^{4}$, donde el silencio y la falta de visibilidad son casi absolutas. Aunque tres películas recientes abordan la orientación sexual y la vejez: En la ciudad sin limites $(2002)^{5}$, A mi madre le gustan las mujeres, y 80 egunean, tan solo las dos últimas nos presentan la relación en el presente y como foco narrativo; mientras que ninguna muestra la intimidad sexual de sus protagonistas.

\footnotetext{
${ }^{4}$ En los últimos años los análisis académicos en torno al cine, la escritura y la cultura queer en España de Alberto Mira, Chris Perriam, Paul Julian Smith, Jill Robbins, Gema Pérez-Sánchez, Tatjana Pavlovic o Santiago Fouz-Hernández dan buena cuenta de la importancia de las expresiones culturales LGBT en la España contemporánea.

${ }^{5}$ Es un thriller dirigido por Antonio Hernández en el que Víctor llega a París con su novia para visitar a su padre, Max, gravemente enfermo. . Al llegar se encuentra que el negocio familiar va a ser vendido y que su padre le pide desesperadamente que le ayude a encontrar a alguien. . Ese alguien es el hombre con el que Max tuvo una relación en el pasado y al que traicionó durante los años de la resistencia al franquismo en el exilio francés.
} 
80 egunean fue recibida de manera más o menos positiva por la crítica, la cual alababa la labor de las actrices y la valentía por tratar el tema de los sentimientos y emociones de dos mujeres mayores en relación a la identidad sexualidad. El filme consiguió varios premios en festivales, particularmente de cine LGBT --Hamburg Queer Film Festival (Alemania): Globala award (Premio del público); Amsterdam LGTB film Festival (Holanda): Premio del público (mujeres); Amsterdam LGTB film Festival (Holanda): Premio del jurado senior--. Sin embargo, algunos críticos se hicieron eco de elementos que rompían con la fuerza inicial de la historia romántica y melodramática de estas dos mujeres, situando esta ruptura en dos cuestiones: que se trataba de una historia real ocurrida a unas amigas de los directores más jóvenes que las dos mujeres protagonistas de esta cinta; y que, además, a partir del encuentro amoroso la película flojeaba al ofrecer un final decepcionante en el que ni siquiera la amistad era posible (Ocaña 2010).

En 80 egunean, según reza la sinopsis que aparece en la página de internet de la película,

Axun es una mujer de 70 años que acude al hospital para cuidar al ex marido de su hija. Su sorpresa será mayúscula al darse cuenta que la mujer que cuida al enfermo de al lado es Maite, gran amiga de la adolescencia. Pronto serán conscientes de que la química de la relación sigue intacta, igual que 50 años atrás. Las dos se divierten y disfrutan del encuentro hasta que Axun descubre que Maite es lesbiana. Axun tendrá que lidiar con sus sentimientos enfrentados: ¿A quién escuchar? ¿Al corazón o a la razón? (Anon. n.d.)

Maite es profesora de piano en una escuela de música en Donostia que está a punto de jubilarse; por el contrario, Axun es una mujer de 70 años, ama de casa y 
madre, que vive una vida aburrida en un caserío con su marido, Juan Mari. El silencio y el aburrimiento son las notas dominantes en la relación de Axun con su marido; a la vez que el desprecio es lo que recibe de su hija, Josune, quien vive en EE.UU. Junto a estos personajes, Julián, director de la escuela de música, aunque conoce la orientación sexual de Maite, muestra calladamente en la película que está enamorado de ella. Asimismo, el personaje joven de la película es Garazi, sobrina de Axun y Juan Mari, quien estudia medicina y hace las prácticas en un hospital de Donostia. Por último, se nos presentan a dos hombres en coma: el hermano de Maite y el ex marido de Josune, Mikel.

Desde las escenas iniciales, la película se construye a base de contrastes, de binarismos que afectan tanto a los personajes protagonistas como a los espacios a los que pertenecen. De este modo, la cinta claramente expone el contraste entre los espacios en los que tiene lugar: el rural y el urbano. El espacio rural de Axun y Juan Mari--con su caserío, su bar, su ermita y su residencia de ancianos donde Axun y sus amigas bailan pasodobles con mujeres-- es un lugar en el que la temporalidad parece haberse quedado congelada en el pasado y manifiesta el envejecimiento de su población y la presencia de la Iglesia Católica como institución. En el espacio urbano de Donostia, por el contrario, se ubican el hospital, el teatro, la escuela de música, el banco, etc.; es decir, es un espacio en el que la modernidad y el progreso se manifiestan en sus instituciones. Sin embargo, no se trata solamente de que los dos espacios sean contrapuestos, sino que dentro de ellos los espacios del "hogar" -el caserío de Axun y el piso en Donostia de Maite—se representan de manera diametralmente opuesta. El caserío es viejo y oscuro, y su centro neurálgico es la cocina-comedor, aunque también aparecen el dormitorio, en el que Axun esconde dinero y esconde su batalla identitaria interior, y el granero, donde Juan Mari trabaja 
en sus manualidades. Por su parte, el piso de Maite es amplio, luminoso, bien decorado; el comedor y el salón son los espacios centrales, mientras que el dormitorio y la cocina nunca aparecen. Si las cenas con Juan Mari vienen representadas por el silencio y la incomunicación que hay entre la pareja, los encuentros de Axun y Maite en el comedor de la casa de Maite, hacen que la Axun sometida por Juan Mari y Josune se transforme en una mujer jovial y alegre. En este sentido, estos espacios interiores del hogar serán los que simbólicamente definan a estas dos mujeres.

También a base de contrastes u opuestos funciona la caracterización de cada una de estas dos mujeres y su construcción a lo largo de la narración cinematográfica. De ahí que, aunque las dos mujeres llevan el pelo corto y pantalones, sus abalorios y sus aficiones las distinguen: el punto de cruz de Axun frente a la bisutería juvenil y artesanal que hace Maite, pero que nunca usa; la ropa casi masculina de Maite frente a los pendientes y collares de perlas y el pañuelo de seda anudado al cuello de Axun; el refinamiento y conocimiento de Maite frente a la ignorancia de Axun. Puede afirmarse, por ende, que tanto Maite y su estilo de vestir como su casa son espacios en los que se inscriben la identidad de género y de la sexualidad. Judith Halbertam (2005) define los espacios queer como aquellos en los que la tolerancia hacia la diferencia y la ambigüedad reinan. Se podría argumentar que Maite nos adentra como espectadores a espacios queer; sin embargo, es fundamental observar que, a pesar de que Donostia aparece como una espacio en el que el Otro diferente es tolerado e incorporado, éste sigue siendo un espacio claramente normativo al estar representado, salvo en el piso de Maite, por las instituciones heteronormativas y controladoras como la sanidad, la cultura, la educación, etc., que construyen y sustentan las cuestiones de género y regulan y reprimen la sexualidad (Foucault 1988). Es más, la omisión 
absoluta de un espacio social queer en el que emplazar la identidad de Maite, provoca que la película verdaderamente despliegue una mirada herteronormativa con la que se vela y esconde directamente la diferencia. Si según Liggett y Perry (1995: 2) el espacio físico es fundamental para constituir, mantener y retar la vida social, nada en la Donostia de 80 egunean nos emplaza a la existencia de ese espacio que sitúe a Maite dentro de un grupo o en el que ideológicamente se discuta la pertenencia a este grupo. Al contrario, la soledad y el aislamiento caracterizan a Maite, con lo que se diría que esta reducción y silenciamiento de la identidad lesbiana (Sedgiwck 1990; Mira 2010) dentro de la (post)modernidad urbana de Donostia implica una mirada heteronormativa que expone la sexualidad e identidad de género de Maite como una excepción, una curiosidad más. La ausencia total de espacios sociales para/de/con lesbianas o gais, bisexuales o transexuales, en claro contraste con la presencia de espacios institucionales -el hospital, el banco, el teatro, etc.- - remite a un espacio completamente heteronormativo que no refleja un cambio social cualitativo importante ni para el colectivo LGBT en general, ni para la mujer lesbiana mayor en particular. Maite ha tenido relaciones sentimentales estables, tiene una profesión, es admirada como pianista, es deseada en silencio por un hombre y, además, su identidad lesbiana es considerada por la nuevas generaciones como algo que ya no se esconde, como le explica Josune a Axun. Sin embargo, Maite se nos representa sola, aislada, diferente al grupo y encerrada en el armario simbólico en el que se convierte su casa. De esta manera, Maite se nos muestra como un personaje cuyo género se describe por su contraste con lo que "no es" femenino" y lo que "no es masculino"; es decir, por lo que no la define ni como femenina ni como masculina; por esa doble negación de la que habla Halberstman (1998) que implica el no ser "hombre" aunque adopte ciertos rasgos masculinos, ni ser "femenina" siendo mujer. Es decir, la 
heterosexualidad como imposición patriarcal que Adrienne Rich (1980) analiza, y que el franquismo se aseguró institucionalizar y naturalizar férreamente, es el eje también de esta sociedad vasca que se nos presenta en la película.

El descubrimiento de la identidad sexual de Maite supone un punto de inflexión en la propia identidad sexual y de género de Axun. Al margen de la problematización nostálgica del tiempo perdido, aspecto que se tratará más adelante, y de la recuperación de la juventud a través del deseo, la película esconde tras el binomio corazón (relación lesbiana)/razón (relación heterosexual) presentado en la sinopsis, la identificación generalizada que se hace de las experiencias LGBT con la identidad sexual. Si Judith Butler (1990) expone que el origen de la heterosexualidad patriarcal viene determinada por la asociación que se hace del binarismo biológico varón/hembra con el de géneros hombre/mujer, la identificación realizada en la película de las experiencias LGBT con la identidad sexual desplaza, por lo tanto, las cuestiones de género que también afectan de manera diversa a los hombres y mujeres homosexuales, tal y como ha estudiado Brian Heaphy (2007). De esta manera, la realidad social y de género de Maite se hace casi inexistente en la película, enfocándose la historia y la cámara casi enteramente en su identidad sexual, así como en el dilema y experiencia de Axun, quien al final sigue las pautas heteronormativas de la razón y no de su deseo. Es dentro de este contexto como hay que entender la masculinidad que caracteriza a Maite en su aspecto físico. No únicamente se trata de restablecer heteronormativamente el binarismo hombre/mujer y lo que Maite no es con respecto a las dos partes del binomio, sino de subrayar esa masculinidad como marcador de la identidad lesbiana que imita pero no es original, no es masculina. La homogeneización del lesbianismo bajo esta (no) masculinidad, tal y como ha estudiado Raquel Platero Méndez (2009), fue desarrollada por el franquismo y 
continúa, a pesar de los grandes avances de la sociedad española actual, "la asociación entre trasgresión de normas y patología” (36), manteniéndose así el rechazo a la masculinidad femenina.

En este sentido, en Female Masculinity (1998) Halberstman exploraba cómo la masculinidad femenina ha sido considerada desde diversas perspectivas, heteronormativas o no, como algo o bien negativo o bien positivo; como la expresión de la prevalencia del patriarcado o como la de una alternativa saludable de lo que se considera la feminidad normativa (9). Es decir, la multiplicidad de perspectivas sobre la masculinidad femenina, incluida la patológica anteriormente indicada por Platero Méndez (2009), ponen de manifiesto, siguiendo la línea trazada por la superioridad del poder masculino y no su subversión (Halberstman 1998: 9). Precisamente es esta lectura la que también se puede hacer de la masculinidad de Maite tal y como está (re)tratada en 80 egunean. Maite cuida de su hermano, pero no del mismo modo que lo haría una mujer como Axun, sino que le hace una fiesta de cumpleaños, llenando la habitación del hospital de globos y poniéndoles a los dos enfermos en coma sendos capiruchos de cartón; o haciendo pendientes juveniles y hippies aun cuando tiene 70 años y ella no los usa; o utilizando ropa masculina en vez de femenina. Es decir, en la línea de lo analizado por Halberstman (1998), Maite aparece primero como "no mujer" y posteriormente como "no hombre". No se puede decir que Maite sea representada de manera estereotípica como "marimacho", "camionera" o "tortillera"; todo lo contrario, su masculinidad se fija en lo superficial para dejar intacto su carácter tierno y sentimental asociado con lo femenino, mientras que cada vez con más fuerza se la opone a la fuerza de carácter y egoísmo de Juan Mari.

Sin embargo, hay cuatro momentos en los que lo que prevalece es su masculinidad; pero una masculinidad adoptada que le concede un aparente poder. 
Estos cuatro momentos son el baile y beso que se nos presentan a través de la analepsis-recuerdo de Maite; la escena en la Isla de Santa Clara en la que su papel en la performance es la del hombre seductor; la escena del abrazo en la que es Maite la que toma la iniciativa; y, finalmente, la última escena de la película en la que una Maite más masculina que antes se muestra fría y distante ante el encuentro fortuito con Axun. En estos cuatro momentos, Maite ejerce el papel socialmente asignado al hombre en las relaciones de pareja heterosexuales. Como tal, su masculinidad en el primer momento viene determinada no solamente por el traje de su padre que se pone, sino también por ser quien conduce a Axun en el baile, y por ser quien toma la iniciativa del beso. Del mismo modo ocurre en la Isla de Santa Clara, donde Maite toma la iniciativa del beso pero ahora sin el traje de su padre, por lo que Axun ya no responde riéndose sino enfadada dado que la mascarada que pensó era lo que ocurrió en la adolescencia se ha desvelado. También es Maite quien inicia el abrazo y quien, años después de haber sido rechazada por Axun, se resiste a volver a retomar una posible amistad. Por lo tanto, Maite logra en la narración cierto empoderamiento masculino, pero éste se muestra como usurpación ya que solamente puede explorarse dentro de unos espacios privados que, a todas luces, parecen reflejar las actitudes de género supuestamente masculinas en las relaciones de pareja heterosexuales. Es decir, el imaginario fílmico no propone un espacio queer, sino uno heteronormativo en el que se proyecta el poder masculino en las relaciones de pareja, ya sean del signo que sean.

Más aún, Axun es un sujeto social educado de manera heteronormativa, con papeles de género construidos cultural y socialmente (Butler 1990): el ama de casa, la esposa, la madre, la tía, la cuidadora, la enfermera, etc. Aunque con una experiencia un tanto negativa de la maternidad, su sexualidad es definida como reproductiva, y 
por lo tanto ha sido sometida al orden patriarcal (Foucault 1988). Sin embargo, su edad implica que su capacidad reproductiva ya no defina su identidad; su estado “postmenopáusico” regula y medicaliza su sexualidad (Woodward 1999), convirtiéndola en un sujeto "asexual", tal y como la ausencia de cualquier alusión a una relación sexual con Juan Mari claramente indica. Frente a ella Maite se nos presenta con un deseo sexual todavía activo, alegre, lleva de vida, con una actividad profesional, pero sola. Su identidad viene marcada por la soledad provocada por su orientación sexual, la cual la discrimina y margina socialmente. Pero también viene determinada por estar a punto de dejar de ser sujeto productivo económicamente ante su inminente jubilación. Maite es, en este sentido, un sujeto social triplemente anulado por su identidad de género, su identidad sexual y su edad: su improductividad reproductiva vendrá ahora reforzada por su improductividad económica debido a la edad que la margina y la expulsa del espacio económicamente productivo de la sociedad.

En relación a este posicionamiento no normativo definido desde la heteronormatividad de la cultura a través de binarismos, Maria T. Brown (2009) ha analizado cómo, al igual que la gerontología feminista y la gerontología cultural han silenciado las experiencias de envejecimiento no heterosexuales, se han silenciado las cuestiones de la edad en los estudios queer. Este silencio al que se ven sometidos los mayores no heterosexuales por los discursos normativos es el que define al personaje de Maite en la película. La soledad que caracteriza la vida presente de Maite viene determinada por ser lesbiana y por su masculinidad, recreando de este modo la dicotomía patriarcal, esencialista y heteronormativa hombre/mujer en la relación homosexual de la que habla Maria T. Brown (2009). Una dicotomía que por otra parte se ajusta al papel que los miembros de una sociedad tienen dentro las expectativas 
culturales (Ward et al. 2008). Es de esta manera como hay que entender que tras pasar la noche juntas, Axun decida quedarse con Juan Mari y cuidarle hasta su muerte. Es también como hay que comprender que Juan Mari en ningún caso sospeche de que la relación que Axun pueda tener es con una mujer; todo lo contrario, está convencido de que es con Julián, lo que de nuevo "normaliza" la sexualidad de Axun dentro de la dicotomía esencialista hombre/mujer.

En el momento en el que Axun decide no regresar al hospital durante un tiempo tras el incidente en la Isla de Santa Clara, se produce una escena que viene a poner de manifiesto la discriminación social de los mayores, pero sobre todo de los mayores no heterosexuales. La sobrina de Axun, quien trabaja en el hospital y está conversando con Maite en la habitación del hospital, no disimula la "gracia" que le hace conocer por primera vez a una lesbiana de 70 años. Es más, cuando le pregunta Maite sobre cómo es su tía en el pueblo, Garazi responde que "normal; como todas las mujeres de su edad... es muy tradicional”. En esta nueva identificación entre espacio rural y espacio urbano, con la tradición y modernización respectivamente, se enclava también la identidad sexual. Emplazar la identidad sexual a una cuestión binaria que ve la ciudad como el espacio del progreso, incluso sexual, parece claramente una visión bastante homogeneizadora y discriminatoria, ${ }^{6}$ a la vez que enfatiza más la soledad en la que vive Maite.

Si que Juan Mari crea que Axun le es infiel con un hombre supone la (re)normalización de la identidad heterosexual, que Julián siga enamorado de Maite claramente implica poner de manifiesto la asunción patriarcal de que la orientación sexual de las lesbianas puede ser "rectificada" por un hombre. Por lo tanto, es cuando

\footnotetext{
${ }^{6}$ Una película anterior, Ander (2009), muestra la posibilidad del espacio rural vasco como espacio homosexual; sin embargo, la relación homosexual se da entre dos hombres adultos, no mayores. .
} 
Julián descubre que Maite le ha mentido para ir a la isla con Axun en vez de ir a trabajar, cuando finalmente se da cuenta de que su masculinidad no va a cambiar la identidad sexual de Maite. Esta actitud, esta concepción de que el lesbianismo es como una especie de enfermedad que un hombre puede "curar" se inscribe dentro de la tradición patriarcal y represiva del franquismo, tal y como han analizado Raquel Platero Méndez (2009), Gemma Pérez Sánchez (2007) y Alberto Mira (2004), y cuya supervivencia queda implícitamente reconocida en 80 egunean. De la misma manera, la presencia de la Iglesia Católica, y su importancia en la zona rural, se enfatiza con el funeral y entierro de Mikel, así como con la ermita a la que acude Axun cuando empieza a dudar de sus sentimientos hacia Maite. Esa misma Iglesia Católica, tanto en el País Vasco como en España, fue la ejecutora de una represión sexual ya conocida y ampliamente documentada. Si Bauman (1995: 135) enfatizaba el empleo de la metáfora de la familia como nación, como espacio seguro desde el que se percibe al Otro, Axun elegirá este espacio seguro de la familia porque en él se puede definir de manera esencialista, fija y no fluida. ${ }^{7}$

En cuanto a la temporalidad, la película se ordena cronológicamente en torno a ciertos días que estructuran la narración: desde el encuentro en el hospital hasta el día ochenta en que se ven por última vez--reminiscente del título de La vuelta al mundo en 80 días, de Julio Verne-y, como epílogo, el día 974 cuando se encuentran por casualidad, pero solamente para certificar que incluso su posible amistad ha terminado. Esta cronología tan marcada, así como el salto temporal enorme en el epílogo, contribuye a la construcción de un tiempo normativo que es cíclico, que

\footnotetext{
${ }^{7}$ Aquí nuevamente podría llevarse a cabo un análisis desde la interacción de género, sexualidad y nacionalismo.
} 
termina en el mismo lugar en el que empieza, por lo que no puede clasificarse como tiempo queer, tal y como Halberstam explica, en el que queer subcultures produce alternative temporalities by allowing their participants to believe that their futures can be imagined according to logics of life experience — namely birth marriage, reproduction, and death. (2005: 2)

En efecto, debido a la discriminación social por la edad de sus personajes, la película presenta una temporalidad en la que lo único que resta como experiencia vital es la muerte. Ese paréntesis de ochenta días en el que parece que la temporalidad se hubiera suspendido, o mejor dicho, hubiera recuperado el tiempo perdido, no es más que un regreso nostálgico al pasado que no puede recuperarse por el carácter cronológico del tiempo y el carácter de construcción social de la identidad de género y de la edad. Es decir, nada ha cambiado tras esos ochenta días, todo sigue igual, con lo que este paréntesis en la vida de estas dos mujeres viene a reincidir en la imposibilidad de cambio tanto en el tiempo como en el espacio. Una relación abierta entre dos mujeres mayores no parece posible; no solamente por el aparato social que la reprime, sino porque la mujer misma, como Axun, ha asimilado de manera inquebrantable las cargas sociales impuestas y la construcción como sujeto social de la mujer: la masculinidad reside en el hombre y la feminidad en la mujer. No hay medias tintas como las de Maite; en la vejez lo que queda es cuidar del esposo hasta que la muerte les separe. Tanto la conversación entre Garazi y Maite en la que el lesbianismo en los mayores se ve como algo asombroso, la conversación entre Josune y Axun en el que la propia Axun piensa que el lesbianismo es una moda entre los jóvenes, la creencia de Juan Mari de que Maite está teniendo una relación extramatrimonial con un hombre, así como la esperanza callada de Julián de que 
Maite pueda de pronto cambiar su identidad sexual, provocan a nivel de género y de sexualidad una clara discriminación de los mayores, a quienes se les deniega la posibilidad de cambio.

La identidad sexual y el deseo sexual son dos aspectos que normalmente los discursos hegemónicos patriarcales omiten cuando los sujetos protagonistas son los mayores. De esta manera, se crea la ilusión de que el envejecimiento supone la desaparición del deseo sexual, sobre todo en las mujeres a partir de la menopausia. Por el contrario, Simone de Beauvoir (1983) expone cómo el deseo, a pesar de lo que la tradición tanto médica como filosófica hayan querido retratar, pervive con fuerza en las mujeres tras la menopausia. Es más, la escritora francesa llega a afirmar que la menopausia corta la vida de la mujer en dos, haciéndola discontinua y, por lo tanto, dando la impresión de que la mujer entra en una nueva vida. Es decir, aunque el cuerpo envejezca nuestra sexualidad no lo hace a no ser que hayamos asimilado el discurso hegemónico de que el envejecimiento del cuerpo va acompañado de la decadencia del individuo como sujeto y de su sexualidad. Cuando aproximaciones sociológicas contemporáneas como las de Marshall y Katz (2002) sugieren que una persona no deja de tener relaciones sexuales por la edad, pero que dejar de tenerlas es lo que envejece al individuo, en realidad lo que estas aproximaciones hacen es perpetuar una percepción patriarcal y hegemónica tanto de la vejez como de la sexualidad. La sexualidad es entendida aquí, como señala Gullette (2013: 141-43), como aquella de la juventud, donde la actividad y el orgasmo son sus señas de identidad; de ahí que Gullette abogue por entender la sexualidad como algo que envuelve cualquier expresión de deseo más allá del orgasmo o la penetración.

80 egunean, aunque nos presenta el deseo homosexual en la vejez, sigue sin embargo planteándolo en términos patriarcales y normativos. Si bien es cierto que los 
tópicos del cine y la literatura convencionales no se dan en esta película en tanto en cuanto no se plantean ni la necesidad de parecer más joven, ni de desear a mujeres/hombres más jóvenes_-como es el caso en A mi madre le gustan las mujeres — sí hay dos cuestiones que afectan de manera fundamental a la película: la omisión visual de la relación sexual y el uso de la temporalidad. ${ }^{8}$ Jill Robbins (2003) afirma que la "alta cultura lesbiana" en España no nos muestra una identidad lesbiana estable, a la vez que presenta un erotismo lésbico que, siguiendo la lógica del mercado, todavía sitúa el cuerpo femenino como centro, en consonancia con la dominante heteronormatividad masculina de la esfera pública (116). Es precisamente esta centralidad del cuerpo femenino desde la mirada masculina dominante la que también determina que los cuerpos envejecidos no sean eróticamente atrayentes y, por lo tanto, no merezcan ser mirados ni deseados. La escena en la que Axun cena en casa de Maite y llama a Juan Mari mintiéndole, supone la suma de dos miradas masculinas: la de Juan Mari pensado que Axun tiene una relación con un hombre y la de la cámara que con primerísimos planos de las cabezas de las dos mujeres en el acto de abrazarse en el sofá. Un abrazo del cual la cámara nos muestra la mano de Axun acariciando tímidamente la espalda de Maite; es decir, nos muestra un simple y emotivo abrazo que cesa con un corte seco para pasar a la mañana siguiente.

Es imprescindible en este punto considerar en detalle la analepsis fílmica que supone el recuerdo del beso adolescente. Este recuerdo se produce en dos momentos: al final del día 16 y desde el recuerdo de Axun; y en la escena de la Isla de Santa Clara en el día 34, brevemente recordado por Maite y recreado en el presente con el beso en la isla. Pero si en la juventud al beso le suceden las risas de ambas, ahora lo

\footnotetext{
${ }^{8}$ No es el caso en películas como la de Julio Medem Habitación en Roma (2009) donde las amantes son jóvenes.
} 
que provoca es el deseo de Axun por marcharse de la isla. En la analapesis de Axun, la duración es mayor que en la segunda de Maite, ya que esta última ofrece únicamente el beso. En la primera analepsis la cámara nos presenta la escena contextualizada espacialmente en la casa grande y luminosa de los padres de Maite: Maite sale del cuarto de baño vestida de hombre con el traje de su padre que le queda grande, mientras que Axun está esperándola en la habitación de pie con un vestido blanco y unas trenzas con lazos también blancos; es decir, como típica muchacha burguesa. Las escena, por tanto, se nos presenta con dos personajes heteronormativos a través del enmascaramiento que supone vestir el traje del padre, en un ambiente además completamente idealizado a través de la luz y las risas de las chicas. Es importante enfatizar que, aunque la sinopsis nos dice que esta escena tuvo lugar cincuenta años antes, es evidente que hay una clara incongruencia cronológica, si no anacronismo, al presentársenos dos chicas bastante adolescentes que no parecieran tener veinte años sino más bien unos quince o dieciséis. Se trata, sin lugar a dudas, de un dato importante ya que podría situarse en esta edad, según Halberstam (1998: 6) el punto en el que la masculinidad femenina debe ser reorientada y reprimida para conformarla a las formas de feminidad normativa. Si la escena claramente presenta los papeles de género asignados visualmente a través del vestido y de los roles asignados en términos hombre/mujer, conquistador/conquistada, el regreso imaginario de Axun a esa escena al saber que Maite es lesbiana pone de manifiesto su cuestionamiento de cómo ha desarrollado los papeles de género de madre y esposa socialmente construidos y asignados debido a su condición biológica.

En el caso de Maite, estos ochenta días son un paréntesis que viene a ratificar el orden anterior y posterior; un paréntesis reforzado por la imposibilidad que encuentra Maite de mantener relaciones sentimentales duraderas en la sociedad en la 
que vive, aunque sea en una Donostia modernizada. En el diálogo que precede al abrazo entre Maite y Axun, Maite le explica a Axun quién es la mujer que aparece con ella en una fotografía, su mejor amante, una mujer sueca a la que conoció en París y se vino a vivir con ella, pero que luego se marchó cansada de tener que esconderse. Para Maite la situación se repetirá con Axun: la dejará, pero no únicamente porque haya que esconderse, sino porque su "deber" como "mujer" no se lo permite. Es decir, las experiencias se repiten, no hay avances, no hay cambios; hay solamente repeticiones y calcos. Aquí no hay una temporalidad queer alternativa a los tiempos cronológicos burgueses como la definida por Halberstam (2005); no hay un tiempo postmoderno más allá de las convenciones lineales de las instituciones familiares y de la reproducción biológica. Para Axun este paréntesis es la vivencia de la (segunda) adolescencia en la que la confusión identitaria reina y los límites entre el deseo y la amistad parecen quebrantarse. Es más, la clara ausencia de la posibilidad de una identidad bisexual de Axun en esta película impide radicalmente la existencia de una temporalidad y de un espacio verdaderamente queer, tal y como lo entiende Hemmings (2002), en el que se desestabilice finalmente el binomio normativo hetero/homo.

Por último, es imprescindible examinar el personaje de Josune, la hija única de Axun y Juan Mari. Josune es una mujer ya madura, seguramente en los 40, que vive en EE.UU. De ella el espectador tan solamente sabrá que estuvo casada con Mikel, el hombre en coma en el hospital al que Axun cuida. Hasta la muerte de Mikel, Josune permanece en EE.UU, con lo que conocemos su personalidad a través de las conversaciones telefónicas que mantiene con su madre. Estas conversaciones, al igual que el lenguaje corporal, nos muestran a una Josune dura, déspota con su madre, muy masculina en su manera de actuar. Sin embargo, en Josune, al contrario que en Maite, 
la masculinidad le viene asignada por su carácter duro y represor de la ingenuidad de la madre. Es más, si Marsha Kinder (1993) veía en esas mujeres fálicas y castradoras que poblaban parte del cine de autor español de los 70 y 80 una representación del autoritarismo patriarcal que dominaba durante el franquismo, podría afirmarse que en 80 egunean ese matriarcado castrador se ha desplazado a la hija, quien domina desde la distancia la vida de la madre ${ }^{9}$. Interesa enfatizar en este punto que, a pesar de que se desplaza ese autoritarismo castrante a la hija, se sigue manteniendo el autoritarismo en el sujeto femenino. Es más, si en películas como La muerte de Mikel, como indica también Kinder (1993), la figura del padre está ausente, lo mismo sucede en esta película: Juan Mari, aunque real y presente, no cruza ni una sola palabra con su hija en toda la película; incluso el silencio con su mujer es el que prevalece en casi todas las escenas. Más aún, como se indicó al comienzo, de los otros tres personajes masculinos en la película, dos están en coma y otro sin atreverse a expresar sus sentimiento hacia Maite. Por consiguiente, puede concluirse que lo masculino tiene una presencia fantasmagórica todavía, mientras que el matriarcado prevalece, pero ahora no en la madre sino en la hija. De hecho habría que estudiar esta relación madre-hija desde la perspectiva de Luce Irigaray (1985) y su concepción de que es el hombre el que interviene en el deterioro de las relaciones entre madres e hijas porque son ellos los que controlan tradicionalmente el papel de género asignado a las mismas: esposas e hijas. De la misma manera, Adrienne Rich indicó que 'The "childless woman" and the "mother" are a false polarity, which has served the institutions both of motherhood and heterosexuality' (1986: 250); idea que complementa a la de Judith Arcana de que la opresión de las mujeres ha abierto una gran brecha entre las mujeres, especialmente entre madres e hijas, ya que no se puede

${ }^{9}$ La analepsis lleva al espectador a unos 55 o 50 años atrás; es decir, a la segunda mitad de la década de los 50 del siglo XX, en plena época de represión franquista. 
respetar a las madres en una sociedad que las denigra; es decir, las mujeres no pueden respetarse a sí mismas (1979:1). Mikel, el ex marido de Josune, al que Axun decide enterrar en el panteón familiar, es el fantasma que (des)une a madre e hija. El espacio que ocupa Mikel en cada una de estas mujeres — el marido de su hija versus el más que probable maltratador de Josune — es precisamente el que abre la brecha tanto física como emocional entre madre e hija. Axun encuentra en los cuidados de Mikel el restablecimiento indirecto de la relación casi inexistente con su hija; mientras que Josune encuentra en estos cuidados la perpetuación de un sistema patriarcal en la que el hombre opresor/maltratador sigue ejerciendo su poder y violencia incluso en coma o muerto. Tal vez por esta razón Josune sea representada en la película como una mujer masculina, fuerte de carácter y con un semblante áspero y seco; mostrando al espectador, por ende, otro lado de la masculinidad que pervive en la mujer madura en tanto sujeto social cuando está sola, sin un hombre.

Como se ha observado, Axun es el eje de las relaciones entre los personajes, definiendo y definiéndose en y contra cada una de ellas. Mientras que en su relación con Juan Mari, Josune, Garazi, e incluso Mikel , Axun se define en su identidad de género, es en su relación con Maite cuando su identidad se ve de pronto debatida . Sin embargo, en una clara referencia a la represión sexual y, sobre todo, a la represión sexual en la vejez, ese posible cambio en la identidad sexual de Axun regresa a la normalidad para ser subyugada por su identidad de género socialmente construida. Esto es, si para Braidotti (1994) el proyecto de la diferencia sexual parte de la voluntad política de afirmar la experiencia corporal de la mujer como nuevo sujeto supuestamente "posmoderno" y "antiesencialista", con lo que diferencia sexual conlleva la voluntad de reconectar todo el debate sobre la diferencia con la existencia corporal y la experiencia de las mujeres, con Axun se presenta su fracaso absoluto. 
Por otra parte, se nos presenta a Maite únicamente en su relación con Axun, con lo que es su identidad sexual lesbiana en el ámbito privado, y no su identidad social, lo que interesa o forma el eje de la película. Por eso, los espacios queer no aparecen y, por esta razón, la temporalidad queer tampoco.

En conclusión, aunque de temática queer, 80 egunean nos plantea todavía la mirada heteronormativa a una mujer de setenta años que de manera nostálgica vive un paréntesis vital con su amiga de la juventud. El paréntesis la aleja momentáneamente del tedio de su vida diaria en el caserío, pero no es más que un paréntesis que confirma el triunfo de la heteronormatividad . La película nos ofrece mirada simplista y masculina del lesbianismo y de lo queer, despojándolos de la complejidad inherente a las identidades sexual y de género. Tal y como subraya Alberto Mira en cuanto a la representación de relaciones lésbicas en televisión, 80 egunean también se adhiere a las tres reglas de

a) no mostrar sexo entre mujeres a no ser que se hiciera a través de la mirada de un varón heterosexual b) la relación lésbica era marginal, episódica c) la relación lésbica se presentaba como parte de un triángulo bisexual y en general la clausura determinaba cierto triunfo de la heterosexualidad. (2010: 10)

De esta manera, el paréntesis en la vida de Axun viene a confirmar la imposibilidad de cambio en los mayores, la negación de su sexualidad; es decir, la discriminación y el estigma social siguen afectando a los mayores y, especialmente, a las mujeres mayores . La aparente mirada "insumisa" (Mira 2008) se torna dentro de la representación cinematográfica de 80 egunean en una mirada nuevamente misógina. 


\section{WORKS CITED}

80 egunean, 2011. Jon Garaño and Jose Mari Goenaga. (Moriarti Produkzioak)

A mi madre le gustan las mujeres, 2002. Dir. Daniela Féjerman and Inés París,

(Fernando Colomo Producciones Cinematográficas S.L.).

Ander, 2009. Dir. Roberto Castón, (Berdindu \&ETB).

Anon., n.d. 'Pressbook' [Online] Available from:

http://www.80egunean.com/descargas/pressbook_cas.pdf Accessed:

24th September 2015.

Arcana, Judith, 1979. Our Mother's Daugthers (Berkeley: Shameless Hussy Press).

Aresti, Nerea, (2014). 'De heroínas viriles a madres de la patria. Las mujeres y el nacionalismo Vasco (1893-1937)', Historia y Política, 31: 238-301.

Bauman, Zigmun, 1995, 'The Stranger Revisited - and Revisiting', Life in Fragments. Essays in Postmodern Morality, (Oxford: Blackwell Publishers):126-38.

Braidotti, Rosi, 1994). Nomadic Subjects: Embodiment and Sexual Difference in Contemporary Feminist Theory, (New York: Columbia University Press).

Brown, Maria T, 2009. 'LGBT Aging and Rhetorical Silence. Sexuality Research and Social Policy', Journal of National Sexuality Research Center, 6 (4): 65-78.

Butler, Judith, 1990. Gender Trouble: Feminism and the Subversion of Identity (New York: Routledge).

Cabré, María Ángeles, 2015. 'Unos Goya muy machos', [Online] Available from: http://blogs.elpais.com/mujeres/2015/02/unos-goya-muy-machos.html [Accessed: 23rd September 2015].

Casper, Monica J. y Moore, Lisa Jean, 2009. Missing Bodies: The Politics of Visibility (New York: New York University Press). 
Chivers, Sally, 2011. The Silvering Screen. (Toronto: University of Toronto Press).

Cole, Thomas, Achenbaum, W.A., Jakobi, P.L, and Kastenbaum, R, 1993. Voices and Visions of Ageing: Toward a Critical Gerontology. (New York: Springer Publishing).

Cruikshank, Margaret, 2008. 'Aging and Identity Politics', Journal of Aging Studies, 22: 147-151.

Davis, Ann, 2009. 'Woman and Hom: Gender and the Theorisation of Basque (National) Cinema', Journal of Spanish Cultural Studies, 10 (3): 359-72.

De Beauvoir, Simone, 1983. La vejez, (Madrid: Edhasa).

De Pablo, Santiago, 2012. The Basque Nation On-Screen. Cinema, Nationalism, and Political Violence. Trans. Robert Forstag, (Reno: Center for Basque Studies, University of Nevada).

En la ciudad sin límites, 2002. Dir. Antonio Hernández, (Zebra Producciones, Icónica, Patagonik Film Group).

Featherstone, Mike and Wernick, Andrew, ed.,1995. Images of Aging: Cultural Representations of Later Life (London: Routledge).

Foucault, Michel, 1988. The History of Sexuality, (New York: Vintage Books).

Gabilondo, Joseba, 1997. 'Masculinity’s Counted Days: Spanish Postnationalism, Masochist Desire, and the Refashioning of Misogyny,' Anuario de cine y literatura en español, 3: 53-72.

---. 2014. 'The National Primal Scene: On Spain's Cinematic Invisibility and the Global Emergence of Basque and Andalusian Cinemas.' [Online] Available from http://basque.criticalstew.org/?p=11102 [Accessed: 23rd September 2015]. 
Gullette, Margaret Morganroth., 2004 Aged by Culture. (Chicago: University of Chicago Press).

---., 2011. Agewise: Fighting the New Ageism in America, (Chicago: University of Chicago Press).

Halberstam, Judith, 1998. Female Masculinity (Durham: Duke University Press).

---., 2005. In a Queer Time and Place: Transgender Bodies, Subcultural Lives, (NY: NYU Press).

Heaphy, Brian, 2007. 'Sexualities, Gender and Ageing', Current Sociology, 55 (2): 192-210.

Hemmings, Clare, 2002. Bisexual Spaces: A Geography of Gender and Sexuality, (New York: Routledge).

Irigaray, Luce, 1985. This Sex Which Is Not One, Trans. Catherine Porter, (New York: Cornell University Press).

Katz, Stephen, 1995. 'Imagining the Life-Span: From Premodern Miracles to Postmodern Fantasies', in Featherstone, Mike and Wernick, Andrew, eds.,1995. Images of Aging: Cultural Representations of Later Life (London: Routledge): 61-75.

Kinder, Marsha, 1993. Blood Cinema. The Reconstruction of National Identity in Spain (Berkeley, CA: University of California).

La vida empieza hoy, 2010. Dir. Laura Mañá, (Productora Oviedo).

Liggett, Helen and Perry, David C. , 1995. 'Spatial Practices: An Introduction', in Spatial Practices, Liggett, Helen and David C. Perry Eds. (London: Sage Publication, Inc.).

Marshall, Barbara and Katz, Stephen, 2002. 'Forever Functional: Sexual Fitness and the Ageing Male Body', Body and Society, 8 (4): 43-70. 
Martín-Estudillo, Luis, 2007. 'E1 hacha en la sangre. Nacionalismo y masculinidad en Vacas, de Julio Medem', Journal of Spanish Cultural Studies, 8, (3): 34155.

Mira, Alberto, 2004. De Sodoma a Chueca: historia cultural de la homosexualidad en España 1914-1990, (Madrid: Egales).

---., 2008. Miradas insumisas: Gays y lesbianas en el cine, (Madrid: Egales).

---., 2010 ‘Después de Ellen: paradigmas de representación lésbica

en las series de televisión actuales', Seminario Mujeres, lesbianismo, normalización y estudios Queer, [Online] Available from https://www.centrodeestudiosandaluces.es/datos/factoriaideas/PN06_10.pdf [Accessed 25th March 2016]

Ocaña, Javier, 2010. 'El armario vasco', El País 20-05-2010 [Online] Available from http://elpais.com/diario/2010/05/21/cine/1274392804_850215.html [Accessed 25 ${ }^{\text {th }}$ September 2015].

Pavlovic, Tatjiana, 2003. Despotic Bodies and Transgressive Bodies. Spanish Culture from Francisco Franco to Jesús Franco, (Albany: State University of New York Press).

Pérez-Sánchez, Gemma, 2007. Queer Transitions in Contemporary Spanish Culture, (Albany: State University of New York Press).

Perriam, Christopher, 2013. Spanish Queer Cinema, (Oxford: Oxford University Press).

Platero Méndez, Raquel, 2009. 'Lesboerotismo y la masculinidad de las mujeres en la España franquista', Bagoas, 3: 15-38.

Rich, Adrienne, 1980. 'Compulsory Heterosexuality and Lesbian Existence', Signs. 5 (4): 631-660. 
---., 1986. Of Woman Born, (New York: Norton \& Company).

Robbins, Jill, 2003. 'The (In)visible Lesbia: The Contradictory Representations of Female Homoeroticism in Contemporary Spain', Latina Lesbian Writers and Artists, 7 (3): 107-31.

---., 2011. Crossing Chueca: Lesbian Literary Culture in Queer Madrid, (Minneapolis: University of Minnesota Press).

Rodríguez, María Pilar, 2002. Mundos en conflicto: aproximaciones al cine vasco de los noventa, (Donostia: Universidad de Deusto).

Rodríguez, María Pilar and Stone, Robert., 2015. Basque Cinema: A Cultural and Political History (London: Tauris World Cinema Series).

Roldán Larreta, Carlos. 1999. El cine del País Vasco: de Ama Lur (1968) a Airbag (1997), (Donostia: Eusko Ikaskuntza).

Sedgwick, Eve, Kosofsky, 1990. Epistemology of the Closet, (New York: Routledge).

Stone, Rob and Jones, Helen, 2004. 'Mapping the Gendered Space of the Basque Country', Studies in European Cinema, 1 (1): 43-55.

Torrado Morales, Susana, 2008. El cine vasco en la bibliografía cinematográfica (1968-2007), (San Sebastián: Universidad de Deusto).

Zunzunegui, Santos, 1985. El cine en el País Vasco, (Bilbao: Bizkaiko Foru Aldundia / Diputación Foral de Vizcaya).

Ward, Richard; Jones, Rebecca; Hughes, Jonathan; Humberstone, Nicola and Pearson, Rosalind, 2008. 'Intersections of Ageing and Sexuality: Accounts from Older People'. Ward, Richard and Bytheway, Bill eds. Researching age and multiple discrimination. The representation of older people in ageing research, (London: The Open University): 45-72. 
Warner, Michael, 1991. 'Introduction: Fear of a Queer Planet', Social Text, 9 (4): 317.

Woodward, Kathleen, 1991. Aging and its Discontents: Freud and Other Fictions, (Bloomington: Indiana University Press).

---. ed.,1999. Figuring Age: Women, Bodies, Generations, (Bloomington and Indianapolis: Indiana University Press). 